\title{
SELF, SISTEMAS Y CULTURA: \\ CÓMO ENTENDER A LA PAREJA QUE NO SE COMPRENDE. DISCUSIÓN DE "LA PERSPECTIVA PSICOANALÍTICA RELACIONAL DE LA TERAPIA DE PAREJA" DE P. A. RINGSTROM
}

\author{
Luis Raimundo Guerra Cid ${ }^{1,2}$ \\ $I P R$, IARPP, IPSA-Levante
}

Una de las muchas paradojas con las que los psicoanalistas históricamente nos hemos encontrado, tiene que ver con que un paciente o una pareja acude a nuestra consulta básicamente porque no comprende qué le ocurre o no sabe cómo cambiar aquello que le perturba. Y digo paradoja porque se nos encarga a nosotros, que somos unos desconocidos, la tarea no sólo de comprenderlo sino de devolvérselo drenado y metabolizado a los pacientes, aquello que Luis Cencillo denominó psicodialysis (1977). Así, lo curioso de esta ardua labor presenta a uno de los protagonistas como alguien que se muestra comprometido aunque desvinculado, en principio, afectivamente además de ser alguien anónimo para los pacientes. Desde esta paradoja tratamos de conocer su mundo subjetivo.

Cuando el tratamiento se realiza junto a una pareja, esta actitud base del terapeuta requiere además del conocimiento de las múltiples variables que se van a poner en juego. Factores que se retroalimentan y que generan un fenómeno complejo. El planteamiento que propone Ringstrom adecua esta circunstancia para que podamos llevar a cabo el tratamiento con las mayores garantías posibles. Esto es porque no sólo propone un modelo de fases sino que además marca los factores básicos que hemos de tener en cuenta. La base para tratar no sólo parejas sino problemas de pareja en sí, viene, en nuestra opinión, desde un triple frente que conecta sólidamente con lo planteado por Ringstrom.

\footnotetext{
${ }^{1}$ Guerra Cid, L.R. (2018). Self, Sistemas y Cultura. Cómo entender a la pareja que no se comprende. Discusión de la "Perspectiva psicoanalítica relacional de la terapia de pareja" de P.A. Ringstrom. Clínica e Investigación Relacional, 12 (1): 78-87. [ISSN 1988-2939] [Recuperado de www.ceir.info ] DOI: 10.21110/19882939.2018.120106

${ }^{2}$ Doctor (con grado de premio extraordinario) y Licenciado en Psicología. Licenciado en Antropología cultural y social. Psicoterapeuta psicoanalista (Especialista en psicoterapia por la EFPA/COP). Especialista Universitario en Medicina psicosomática y psicología de la salud. Miembro del board de IARPP-España, miembro de IARPPInternacional, Miembro titular de IPR. Director del Instituto de Psicoterapia de orientación psicoanalítica y antropología (IPSA-Levante). ipsalevante@gmail.com
} 
1) La cultura en sentido extenso y en su aspecto concreto de retroalimentación en un cuádruple marco que incluye al individuo-familia-sociedad-cultura. Marcos que se contienen unos a otros y que son retroalimentados por distintas variables.

2) La actualización del self. Tomar en cuenta los importantes aspectos de los diferentes estados que el self contiene así como los procesos que de aquí se desprenden: procesos de encaje, reconocimiento mutuo, importancia de tomar en cuenta la terceridad (thirdness Benjamin, 2004-), etc. Además, ha de tomarse en cuenta que se ha de combinar la autorrealización en la pareja con la actualización individual del self. A este respecto, Ringstrom plantea la necesidad de un equilibrio donde se ha de tener una mente propia a la vez que se participa activamente de la relación de pareja.

3) Entender la pareja como un sistema complejo dinámico y no lineal, de variables múltiples las cuales no tienen siempre el mismo valor sino que estas dependen del tipo de pareja construida así como de las cualidades de sus miembros.

\section{Cultura.}

Efectivamente, como señala Ringstrom, el modelo relacional es un modelo de campo e integrativo. Por ello no podemos tratar de observar a la pareja aislada de sus lazos culturales, así el primer elemento a la hora de tener en cuenta las dinámicas de la pareja tiene que ver con el aspecto antropológico social y cultural. Es evidente que no podemos tomar en cuenta a la pareja sin el contexto socio-familiar-cultural del que participa. Una pareja, aparte de ser un sistema, se relaciona con otros sistemas que a la vez interaccionan con ella, la envuelven, fusionan etc. (Guerra, 2013, p 46).

Acertadamente Ringstrom nos hace reflexionar sobre cómo la revolución industrial genera un cambio en la pareja motivada por el amor romántico en su construcción. Pero aquí, precisamente, se nos presenta un escollo de reflexión pues esta época a la que refiere Ringstrom también ha sido denominada como la sociedad "pesada" (Bauman, 2000) contrapuesta a la actual sociedad líquida y no a una postmodernidad anhelada por muchos sociólogos donde la tolerancia y el respeto por lo distinto serían frecuentes. En mi opinión, esto genera una serie de factores que el terapeuta de pareja o el individual que trate problemas amorosos ha de tener en cuenta. Para Bauman la sociedad líquida se basa, entre otros factores, en la globalización y en la comunicación vacua digital. Es interesante pues en muchos casos esto acentúa lo mágico que se fantasea de la relación y lo que es peor, la instrumentalización del otro que anula su reconocimiento. 
A esto le tenemos que añadir lo que consideramos el principio fundamental de la dinámica de la pareja, la idea de formar pareja y la de matrimonio son una construcción cultural, no hay un modo claro de saber cuáles son las funciones que tiene ni cuál ha de ser el comportamiento de sus miembros. Sobre este aspecto las etnografías realizadas en el SXX han arrojado información fundamental.

Por poner sólo algunos ejemplos, tenemos a los Tiv nigerianos, estudiados por Bohanann (1992) donde en el matrimonio se esperaba que la esposa cocinara además de dormir con él y cuidar de los cultivos entre otras tareas. Mientras, él debía vestir a la esposa, preparar la mejor tierra de cultivo o llevarla al chamán si esta se ponía enferma.

Por otra parte, en los Nuer del Sudán, estudiados profundamente por Evans-Pritchard, (1951) vemos que una mujer se puede casar con otra adoptando el papel de varón si su padre no tiene descendientes varones. Así puede presentar a su hija para que se muestre socialmente como hijo y poder tomar una esposa. Esta hija se convertirá en el esposo socialmente reconocido y una de sus funciones fundamentales será seleccionar un varón para su mujer con el que pueda concebir. Este varón será el padre del futuro hijo mientras que el esposo-mujer no tendría dicho rol paterno mientras que mantendría el de cónyuge.

Si eliminamos el mandato socio-cultural, no podemos tener una idea clara de cuáles son las funciones de la pareja y sobre todo de qué factores influyen de modo definitivo como es, entre otros, el de la ideología de género. Pero ¿y cómo vamos a tener claro cuál es la función de matrimonio si ni siquiera hay consenso entre los antropólogos de que la procreación es el factor crucial para que haya parentesco? (Scheneider, 1984, Carsten, 1995). Por otra parte, a partir del primer estudio antropológico y transcultural realizado por W. Jankowiak y E. Fischer (1992), se observa una gran influencia de la globalización y la influencia de occidente y sus mass-media en la idea cada vez más generalizada de amor romántico, lo cual no quiere decir que este tenga que ir en la dirección de la idealización y de pretender reparación, sino más bien del cuidado afectivo esperado por el otro.

Esta cosmovisión del mundo de la pareja nos ofrece una construcción cultural que es fundamental para cualquier terapeuta pues nos hace entrar en el setting a través de un relativismo socio-cultural. Esta actitud nos lleva de lleno a tomar las demandas y reproches de la pareja en perspectiva y como fruto de nuestra construcción cultural. También hará que el terapeuta tenga una disposición activa, abierta, exploradora pero sobre todo diferente a cómo la pareja está acostumbrada a relacionarse y a ser comprendida por el contexto. Digamos que este punto de inicio es la base de la experiencia emocional correctora en terapia de pareja. expresa. Este material es para uso científico y profesional exclusivamente y puede contener información clínica sensible. Los editores no se responsabilizan de los contenidos de los autores. Dirigir las consultas sobre derechos y autorizaciones a ceir@psicoterapiarelacional.es 


\section{Self múltiple a tres bandas.}

Muy pocos han sido los psicoanalistas que académicamente han tratado el tema del amor en la pareja humana. De dos autores clásicos, Reik y Fromm, se traslucen dos ideas bastante interesantes. Para Reik (1946) la idealización es un hecho muy importante en el enamoramiento. Ver en el otro lo que me falta o creo que me falta es un detonante para que el amor se produzca. Por su parte, Fromm (1956) aludía a la complicación de la pareja como ser dos pero siguiendo siendo uno. Rescato estas ideas porque me parecen muy cercanas a lo que Ringstrom comenta y que nos acerca a la complicación de poder actualizar nuestro self y autorrealizarnos en la relación. Señala el autor en su intervención: "Con este compañero, espero ser capaz de actualizar lo que hasta ahora no he podido. Sin embargo, con este compañero, también está el temor a volver a visitar algún aspecto de mi pasado."

Una buena aproximación a la pareja sería verla como una experiencia de crecimiento conjunto que retroalimenta el crecimiento personal (y viceversa) en el que a la vez se pueden conocer otros estados del self propios. Esto puede provocar en cada individuo tener una autoposesión flexible de la identidad (Cencillo, 1998). Este laborioso ejercicio se ha de intentar llevar a cabo con la pareja. Pero hay un escollo a tener en cuenta y es que fruto de las experiencias traumáticas el self se disocia y sus diferentes estados no guardan comunicación unos con otros. Y si la hay se genera una discrepancia de verdades entre ellos que produce ansiedad y por tanto hay una tendencia a fortalecer esa disociación (Bromberg, 2011).

A esto se le añade como problema, que fruto de esos traumas a menudo tenemos atribuciones y expectativas sobre el otro que funcionan desde la compensación de las fallas del self propias y aunque en un principio esta dinámica es una causa de unión y enamoramiento, a la larga pueden convertirse en reproches y en los principales problemas en la cotidianidad de la pareja. Esto ocurre sobre todo si no hay un reconocimiento del otro sino la búsqueda narcisista de la compensación de nuestras carencias en la pareja de modo pasivo y moroso. Las atribuciones y expectativas, que constituyen el armazón que sustenta la idealización (Guerra, 2018b), son un arma de doble filo porque a menudo observamos cómo, la esperanza desprendida de ellas, se convierten en reproche, en el problema, según va avanzando la relación.

Pongamos por ejemplo algo muy observado en las consultas, una persona que sufre un complejo de que va a ser abandonada (aquello que autores clásicos denominaron neurosis de abandono o abandónica, p.e. G. Guex, 1950). Aun con estos temores, puede ceder a sus miedos de emparejarse y situar al otro como un salvador que tiene la misión de mostrar que 
no va a abandonar y que puede compensar "todo". Ante la imposibilidad de que esto ocurra lo ideal que se espera del otro, se transformará en futuras recriminaciones.

Así tenemos que tomar en consideración un complejo interjuego de los estados del self de ambos miembros de la pareja, estados que a menudo no son siquiera conscientes para cada uno de ellos, pero que sin embargo están infraliminalmente activos y jugando su papel. Además, también entran en juego los propios estados del Self del psicoterapeuta, sobre todo los que correlacionen más con la situación analítica que si son disociados provocaran impasse e incomprensión del caso.

Ante toda esta amalgama de factores, necesitamos clarificar los aspectos motivacionales de la pareja y de sus miembros. Por ello es fundamental observar "cómo se manifiestan los deseos y las metas de cada miembro para su autorrealización" ${ }^{1}$. (Ringstrom, 2014, p 51). Tenemos que conocer tanto las necesidades individuales como lo que se espera de la pareja. Esas expectativas que se tienen del otro y del hecho cultural de estar en pareja son más relevantes de lo que a priori parecieran. De hecho, desvelar aquello que cada uno espera de ese vínculo es fundamental para poder, curiosamente, "objetivar" lo que ocurre, a través de un proceso de reconocimiento intersubjetivo. El axioma que propone Ringstrom y que sintetiza estas ideas es "que las parejas no pueden auténticamente negociar entre sí (intersubjetivamente), lo que no han negociado dentro de sí mismos (intrasubjetivamente)".

La negociación intersubjetiva es un fenómeno básico en la pareja, y en esta tesitura compartimos la idea de Coderch y Plaza (2016, p 217) consistente en diferenciar la negociación política de la negociación de comunión. En la primera se puede ver en algunas parejas donde desde una posición de adversarios tratan de obtener beneficios a costa del otro. Pero en la negociación de comunión se busca hacer comunidad, vincularse con el otro y tratar de obtener lo que se necesita sin perjudicar a la pareja. En términos de Alfred Adler diríamos que no sólo el sentimiento de comunidad es algo que da sentido a la vida del individuo, sino que también lo puede hacer en la pareja, pues esta es, ante todo, una experiencia de crecimiento compartido. Y este aprendizaje contempla también las múltiples facetas de la identidad de cada miembro de la pareja.

A través de estas reflexiones, cada vez se nos aclara más que la psicoterapia de pareja es una situación de self múltiple a tres bandas. Hemos de atender a los diversos estados de la pareja y a los nuestros propios. Por ello merece la pena hacer un inciso acerca de estos estados del self que implícita y explícitamente estoy denotando. Podemos concebir la identidad humana como esas muñecas rusas -las matrioskas- que van encajando unas dentro de otras, si tomamos la matrioska grande que contiene a otras, vemos metafóricamente que esta tiene distintos estados representados en cada una de esas muñecas. expresa. Este material es para uso científico y profesional exclusivamente y puede contener información clínica sensible. Los editores no se responsabilizan de los contenidos de los autores. Dirigir las consultas sobre derechos y autorizaciones a ceir@psicoterapiarelacional.es 
En estas matrioskas no solo es importante saber cuál es y dónde está la más pequeña, la cual presuponemos como nuclear y más auténtica, al menos desde la metáfora. Puede que en realidad, tan importante sea esta pequeña muñeca como el resto de matrioskas, puesto que lo nuclear consiste en conocer cómo se combinan estas muñecas para encajar y cómo, dependiendo de las circunstancias, salen a la palestra de las interacciones cotidianas unas matrioskas u otras. Del mismo modo podemos observar cuales constituyen estados dominantes ofreciendo un protagonismo continuado en nuestra vida (Guerra, 2018a, p 47). Estas matrioskas no han de encajar perfectamente unas dentro de las otras, como habitualmente se hace con las muñecas rusas, sino que tal y como ocurre con los estados del self, pueden o no encajar del todo. Así estas matrioskas podrían quedar superpuestas, o si nos imaginamos unas matrioskas construidas con material flexible, con goma por ejemplo, pueden encajar independientemente del tamaño unas con otras, aunque no sea de una manera exacta. Probablemente el self tiene una tendencia más hacia este funcionamiento que hacia otro más ideal de "encajes perfectos".

Philip Bromberg, señala que es básico que en el ser humano haya una comunicación entre los distintos estados del self existentes, las matrioskas han de hablar entre sí. Ello requiere que nuestra identidad haga un bagaje entre la separación de estos estados (con las diversas cualidades de cada una de ellos) y su unidad. Si esto ocurre, aunque tengamos comportamientos o capacidades diferentes en distintos contextos, no lo viviremos como algo ajeno y extraño a nosotros, sino que seguiremos teniendo una sensación de identidad, de «yo» como agente. Dice Bromberg de manera muy acertada que cada estado del self:

[...] tiene su propio grado de acceso a varios dominios de la funcionalidad psíquica (por ejemplo, la capacidad para sentir y tolerar la presión de nuestras necesidades y deseos, la capacidad para juzgar lo que es un comportamiento social adaptativo, la capacidad de amar...). (2011, p 47).

Nuestra identidad y sus diversos estados contienen registros variados de memoria (declarativa y por supuesto procedimental) y otras variables como la identidad de género, orientación sexual, motivaciones, tendencias, etc. Todo ello añade matices muy complejos en la psicoterapia de pareja pues hemos de "lidiar con" y comprender a todas las matrioskas que hay en cada miembro de la pareja, a la vez que contamos con las nuestras como terapeutas. Y nosotros debemos de observar, sobre todo, las que pueden verse disociadas por conectar el conflicto de la pareja tratada con algunos de nuestros posibles escotomas. Las múltiples disposiciones y combinaciones que los estados del self contienen y sobre todo sus disociaciones generarán distintos tipos de enactment. 


\section{La pareja como un sistema dinámico y no lineal.}

Ante todo lo expuesto, vemos una complejidad multivariable en la pareja y en su tratamiento. De este modo se requiere un modelo de aproximación terapéutico que pueda tener en cuenta el mayor número de factores posibles. Cuando se produce una problemática en la relación de pareja esta puede tener una serie de problemas delimitados y "corrientes" (por ejemplo, los roles adoptados en la casa relacionados con la intendencia) o problemas delimitados, "corrientes" y además lo que hemos denominado una afectopatología relacional (Guerra, 2006, 2013) ${ }^{2}$, en la cual vemos un complejo sistema que se autoestructura y en donde las condiciones iniciales y los mantenedores son cruciales.

Resumiéndolo, si entendemos la relación de una pareja como un sistema de estas características, es porque entre los dos protagonistas se halla una enorme diversidad de variables emocionales, comportamentales y motivacionales que influyen sobre el otro, y viceversa. Una relación de pareja es un complejo sistema (dinámico y no lineal) en que las condiciones iniciales y los mantenedores van a producir diferentes modos de relacionarse, algunos de los cuales son absolutamente perniciosos, no sólo para la relación en sí, sino para la salud mental de cada uno de ellos, en especial si cada miembro de la pareja empieza a tener roles fijos del tipo progresivo-regresivo o activo-pasivo.

Desde este foco hemos que tener en cuenta como algo básico las condiciones iniciales en cómo se fragua la relación. Como señala E. Lorenz (1972) la potencia de las variables insertas en las condiciones iniciales de un proceso complejo, pueden provocar fuertes efectos mariposa y cambios impronosticables. Cuestión que no escapa al sistema complejo de la pareja.

Algunos de estos factores a tener en cuenta serían como condiciones iniciales (que a su vez pueden generar atractores dada su sensibilidad dependiente y que se pueden desarrollar a lo largo del proceso de pareja): desfondamiento radical, necesidades de filiación, necesidades de validación y especularización, conocimientos relacionales implícitos y compartidos, atribuciones, expectativas, idealización, tipo de sistema de apego, imprintings, etc. Entre los mantenedores que una relación de pareja puede tener encontramos, aparte de algunos de los atractores que a su vez pueden funcionar como mantenedores: una compensación narcisista de un self desestructurado, el efecto Zeigarnick (con sus consecuentes tendencias repetitivas a buscar el afecto no dado en distintas personas y situaciones), la fobia al abandono, el temor a la soledad, etc. (Guerra 2018b).

Observando estas variables y detectando cuales son las que más influencia tienen en la pareja en concreto, podemos trazar un mapa de intervención para aproximar a ambos protagonistas hacia un proceso de reconocimiento mutuo. Pero estas no son las únicas 
variables a observar en este sistema complejo, también tienen su importancia los efectos acumulativos y los cambios drásticos que en un momento dado se dan. Esa gota que colma el vaso y que en ocasiones es la que trae a la pareja a terapia. También la fractabilidad de las vivencias que la pareja tiene es fundamental.

A este respecto retomo el comienzo de la intervención de nuestro colega americano. Esa secuencia a la que refiere en el setting de la pareja, en la cual ella llega tarde y él se siente mal y que a su vez remite a episodios de abandono por una parte y de oposicionismo por otro, nos colocan ante un fractal. El microproceso reproduce el proceso, muchos de esos episodios que se producen en la terapia, dentro de una sesión, están en realidad replicando procesos que se están produciendo en la pareja. En estas secuencias debemos de intervenir mostrando la actitud de cada miembro, su motivación para llevarla a cabo y por supuesto la reacción generada en el otro. Así sabemos qué significa retrasarse para la esposa y cómo vive el esposo el retraso, todo a partir de sus memorias declarativas y procedimentales. A partir de aquí se puede también expresar lo que se espera y no se espera del otro, en definitiva un proceso de conocimiento propio y reconocimiento del otro.

Cuanta mayor comprensión tengamos de estas variables, más fácilmente podremos encaminar a la pareja hacia un proceso de reconocimiento mutuo, pues todos estos factores se hallan implícitos en la terceridad, el sacar a la pareja del "tú y yo" para incluirlo en el "nosotros", ese lugar en donde está aquello que nos gusta y disgusta del otro, pero también el camino común que lleva a una pareja a ser tal. La rendición contigo y tu rendición conmigo, sin sumisión, sin reproches, "sólo" lo que tú y yo somos y con un conocimiento profundo del otro. Lo contrario de la terceridad es una dialéctica construida entre perseguidor y perseguido, entre víctima y verdugo. De todas las afectopatologías relacionales que hemos detectado la más comúnmente observable es la de rivalidad, una manera de relacionarse donde lo importante es saber quien posee la verdad y quién se equivoca, todo ello a costa de lo que sea. Creo que esta es la mejor definición de "contraterceridad", la cual se puede atajar con la recomendación que hace nuestro invitado de potenciar el reconocimiento a través del cual "comienzan a ver que son lo mismo. Circunstancia que rompe patrones estáticos para dejar de tratarse como "objetos" para tratarse como "sujetos".

\section{Conclusiones.}

Según lo expuesto y dada la complejidad de múltiples factores intervinientes es obvio que para el tratamiento de la pareja necesitamos de un modelo de partida para poder trabajar con los aspectos del Self, las dinámicas no lineales y las continuas retroalimentaciones que se producen en, desde y hacia la pareja. Por ello creo que una propuesta inteligente para 
abordarlo es la utilización de un método. El modelo que Ringstrom $(2012,2014)$ plantea de seis pasos es un método muy adecuado para este propósito. En primer lugar, porque es un modelo abierto e intersubjetivo, lo que nos va a aportar capacidad de maniobra y de poder atender a nuevas variables incluyendo las que sean más idiosincrásicas de esa pareja. En segundo término, porque sigue una estrategia, algo en nuestra opinión absolutamente necesario cuando el volumen de las variables intervinientes pueden poner en jaque nuestra comprensión del caso. Y por supuesto, por la flexibilidad que ya de base poseen los modelos apoyados en las escuelas relacionales. De esta manera a través de los seis pasos propuestos podemos visitar diferentes áreas de estancamiento de la pareja, para dinamizarla y llevar todo a un bien común.

La resultante de todo el trabajo propuesto por Ringstrom es la terceridad, una visión intersubjetiva de los roles, atribuciones y deseos de autorrealización negociados y en donde se ha producido un reconocimiento mutuo. El autor propone que cuando se han trabajado todas las cuestiones previas, esta labor que el terapeuta ha hecho de "el tercero" hace posible que la pareja participe por sí sola de esa terceridad sin la presencia del terapeuta. Esta disposición estaría próxima al espectro de internalizaciones de la figura del terapeuta expuesta por Western y Gabbard (2003) pero enfocado tanto en la internalización individual como en la terceridad de la pareja.

\section{Referencias}

Adler, A. (1930). El sentido de la vida. Madrid, Ahimsa, 2000.

Benjamin, J. (2004). Beyond doer and done to: an intersubjective view of thirdness, The Psychoanalytic Quarterly, 73 (1), 5-46.

Bohannan, P. (1992). Para raros, nosotros. Madrid, Akal, 1996.

Bromberg, P. M. (2011). The shadow of the tsunami and the growth of the relational mind. Nueva York: Routdlege (version en castellano: Bromberg, P. La sombra del tsunami y el desarrollo de la mente relacional. Madrid: Ágora, 2016).

Carsten, J. (1995). The substance of kinship and the heat of the hearth: feeding, personhood, and relatedness among Malays in Pulau Langkawi. American ethnologist, 22:223-241.

Cencillo, L. (1977). Transferencia y sistema de psicoterapia. Madrid: Pirámide.

Cencillo, L. (1998). Labilidad psíquica y terapia dinámica. Madrid: Ediciones Fundación.

Coderch, J. y Plaza, A. (2016). Emoción y relaciones humanas. Madrid: Ágora Relacional.

Evans-Pritchard, E. E. (1951). Kinship and Marriage Among the Nuer. Oxford: Clarendon Press

Fromm, E. (1956). El arte de amar. Barcelona: Paidós, 2000.

(c) Derechos reservados/Copyright de Clínica e investigación Relacional y los autores. Prohibida la reproducción total o parcial sin autorización expresa. Este material es para uso científico y profesional exclusivamente y puede contener información clínica sensible. Los editores no se responsabilizan de los contenidos de los autores. Dirigir las consultas sobre derechos y autorizaciones a ceir@psicoterapiarelacional.es 
Guerra Cid, L.R. (2006). Este no es un libro de autoayuda. Tratado de la suerte, el amor y la felicidad. Bilbao: DDB.

Guerra Cid, L. R. (2013). El clavo ardiendo. Claves de las adicciones amorosas y de los conflictos en las relaciones sanas y patológicas. Barcelona: Octaedro.

Guerra Cid, L.R. (2018a). Palos en las ruedas. Una perspectiva relacional y social sobre por qué el trauma nos impide avanzar. Barcelona: Octaedro.

Guerra Cid, L.R. (2018b). Any port in a storm: a complex and relational focus on addiction in love and relationships, en Ávila, A. (Ed.). Relational horizons Mediterranean voices bring passion and reason to relational psychoanalysis. International Psychoanalytic Books, en prensa.

Guex, G. (1950) The Abandonment Neurosis. London: Karnac Books, 2015

Jankowiak, W. R., Fischer, E. F. (1992). A cross-cultural perspective on romantic love. Ethnology 31(2): 149-156.

Lorenz, E. N. (1972). «Predictability: does the flap of a Butterfly's wings in Brazil set off a Tornado in Texas». American association for the advancement of science in Washington. Washington DC.

Reik, T. (1944). A Psychologist Looks at Love. In M. Sherman (Ed.). Of Love and Lust. (pp. 1194). New York: Jason Aronson.

Ringstrom, P. (2012). A Relational Intersubjective Approach to Conjoint Therapy. International Journal of Psychoanalytic. Self Psych. 7: 85-111.

Ringstrom, P. (2014). A Relational Psychoanalytic Approach to Couples Psychotherapy. London: Routledge.

Schneider, D. (1984). A Critique of the Study of Kinship. Ann Arbor: University of Michigan Press.

Western, D. \& Gabbard, G. (2003). Rethinking therapeutical action. International Journal of Psychoanalysis. 84: 823-841.

NOTAS:

\footnotetext{
${ }^{1}$ La traducción es nuestra.

${ }^{2}$ Esta consiste en un desajuste severo en la gestión de las emociones y los afectos así como una distorsión en la valoración de «dónde» (en qué tipo de personas), «para qué» (qué tipo de fin se persigue) y "cómo" (patrones relacionales implícitos) se emplean los recursos energéticos (físicos y psíquicos) por parte del individuo. Digamos que la afectopatología relacional funciona como un sistema complejo en el cual cada miembro a través de sus propios desajustes mantiene la estructura. Esta circunstancia es fundamental para entender la negación mutua que se produce en muchas parejas alejadas de los procesos de encaje y mutualidad.
} expresa. Este material es para uso científico y profesional exclusivamente y puede contener información clínica sensible. Los editores no se responsabilizan de los contenidos de los autores. Dirigir las consultas sobre derechos y autorizaciones a ceir@psicoterapiarelacional.es 